\section{PSICOLOGÍA IBEROAMERICANA}

\section{Psicología lberoamericana}

ISSN: 1405-0943

psicología.iberoamericana@uia.mx

Universidad Iberoamericana, Ciudad de

México

México

Martínez Pérez, Magali; Retana Franco, Blanca Estela; Sánchez Aragón, Rozzana Identificación de las Estrategias de Regulación Emocional del Miedo en Adultos de la Ciudad de México

Psicología Iberoamericana, vol. 17, núm. 2, julio-diciembre, 2009, pp. 49-59

Universidad Iberoamericana, Ciudad de México

Distrito Federal, México

Disponible en: http://www.redalyc.org/articulo.oa?id=133912609007

Cómo citar el artículo

- Número completo

- Más información del artículo

Página de la revista en redalyc.org

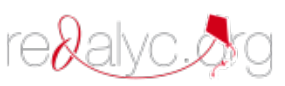

Sistema de Información Científica

Red de Revistas Científicas de América Latina, el Caribe, España y Portugal Proyecto académico sin fines de lucro, desarrollado bajo la iniciativa de acceso abierto 


\title{
Identificación de las Estrategias de Regulación Emocional del Miedo en Adultos de la Ciudad de México
}

\author{
Identification of Strategies for the Emotional Regulation \\ of Fear in Mexico City's Adults
}

\author{
Magali Martínez Pérez \\ Blanca Estela Retana Franco \\ Rozzana Sánchez Aragón \\ Universidad Nacional Autónoma de México, CD. de México, México ${ }^{1}$
}

\section{RESUMEN}

El miedo es una de las emociones que el ser humano vive con mayor intensidad debido a su impacto fisiológico (Lelord, 2001) y a su capacidad de bloqueo emocional, que puede generalizarse a las propias acciones del individuo con lo cual se pierde el valor funcional y adaptativo que posee esta emoción (Berástegi, 2007). Es por ello que su regulación se vuelve relevante. La Regulación Emocional (RE) ha sido segmentada en cinco procesos cognoscitivos por Gross y Thompson (2007), los cuales se operacionalizaron (Sánchez-Aragón, 2008) con el fin de identificar las principales y particulares estragias de RE para el miedo. Para ello se trabajó con 184 adultos mexicanos en quienes se validó la Estimación Cognoscitiva de los Procesos de RE y se encontraron tácticas de enfrentamiento emocional como: alejamiento, alteración del evento, distracción, reflexión-calma, búsqueda de apoyo, evaluación de recursos y agresión -entre otras-.

Descriptores: Emoción, Miedo, Regulación Emocional, Estrategias.

\section{ABSTRACT}

Fear is one of the emotions experienced by the human being as very intense due to its physiological impact (Lelord, 2001) and its capacity to produce in the individual an emotional numb that can be generalized to their own behaviors, which causes to lose the functional and adaptative value of this emotion (Berástegi, 2007). Due to this, its regulation becomes relevant. The Emotional Regulation (RE for its initials in Spanish) has been separated in five cognitive processes by Gross and Thompson (2007), which where operationalized by Sánchez-Aragón (2008) trying to identify the main and specific strategies of RE towards fear. In order to do so, we validated the Cognitive Estimation of ER Processed in 184 Mexican adults and we could find some tactics used to cope with the experience of this emotion: separation, altering the event, distraction, reflection-calm, seek for support, evaluation of resources and aggression, among others.

Keywords: Emotion, Fear, Emotional Regulation, Strategies.

\footnotetext{
${ }^{1}$ Para correspondencia: Magali Martínez Pérez. Dirección postal: 4ta. Cerrada de Juan Escutia S/No., San Salvador Cuauhtenco, Delegación Milpa Alta, C.P. 12300, México, D. F. México. Tel.: (52) (55) 5862 1403, magy_0313@yahoo.com.mx

Blanca Estela Retana Franco, Dirección postal: Kinchil Mz. 80 Lt. 14 Colonia Lomas de Padierna, Delegación Tlalpan. México Distrito Federal. Tel.: (52) (55) 5644 0973, blanca_retana_franco@hotmail.com

Rozzana Sánchez Aragón, Universidad Nacional Autónoma de México. Dirección postal: Chimalcoyotl 14 Casa 4 Col. Toriello Guerra, C.P. 14050 Del. Tlalpan, México, D. F. México. Tel.: (52) (55) 5665 0158, rozzara@servidor.unam.mx
} 


\section{INTRODUCCIÓN}

Las emociones son fenómenos psicológicos que dan a la vida su sabor y sus sinsabores (Reidl-Martínez, 2005). De ahí que uno de los principales intereses del ser humano sea el adentrarse en su estudio para llegar a su cabal entendimiento; labor que no ha sido fácil ya que incluso su definición y tipologías sufren actualmente de controversia. Así, aunque en un inicio la palabra emoción (emovere $=$ moverse hacia fuera) hacía referencia exclusivamente al movimiento, alcambio, al impulso involuntario de hacer algo (Märtin \& Boeck, 1998), progresivamente ha ido dejando atrás su raíz etimológica para integrar a su definición elementos que van desde aspectos fisiológicos hasta aquellos con un tinte social. Bajo esta luz, Sánchez-Aragón (2007) señala que la emoción es un producto de la interacción de sensaciones, pensamientos, conductas y cambios corporales enmarcados en un contexto cultural en particular que los dota de sentido y que regula -entre otros aspectos- su percepción, expresión y entendimiento.

En lo que los estudiosos de las emociones (por ejemplo Ben-Ze'ev, 2000; Reidl-Martínez, 2005) parecen estar de acuerdo es en que éstas poseen características particulares como su espontaneidad, inestabilidad, brevedad o duración limitada e intensidad, aspectos que según Ben-Ze'ev (2000) les han valido para ser igualadas con fenómenos tales como las tempestades o el fuego mismo.
Ahora bien, en lo que a su categorización se refiere, se ha planteado que las emociones pueden agruparse en dos vertientes: positivas y negativas, en función de sus implicaciones en la experiencia subjetiva de éstas, así como en su impacto interpersonal. De esta manera, aquellas que motivan a los individuos a aproximarse al objeto de la emoción son consideradas positivas, mientras que aquellas que motivan a evitarlo entran en el rango de las negativas (Wulkimir, 1967 en Goleman, 1997; Camacho, 2002). Es bajo esta línea de pensamiento que Fischer, Shaver y Carnochan (1990 en Feldman, 2002) plantean una jerarquía de emociones (Figura 1).

Esta clasificación refleja -como señalan Märtin y Boeck (1998)- que las emociones de ambos polos son reacciones útiles que cumplen una función adaptativa y favorecen la autodefensa y la supervivencia, pues si bien las emociones positivas producen bienestar, las negativas conducen al alejamiento.

De entre las emociones referidas, el miedo en particular es considerado como una emoción básica negativa que cuenta con el mayor valor funcional en la supervivencia de una gran variedad de especies a lo largo del proceso evolutivo. Esto se refleja en su permanencia en prácticamente todos los organismos vivos y en la interpretación correcta que éstos hacen de las manifestaciones expresivas de dicha emoción en

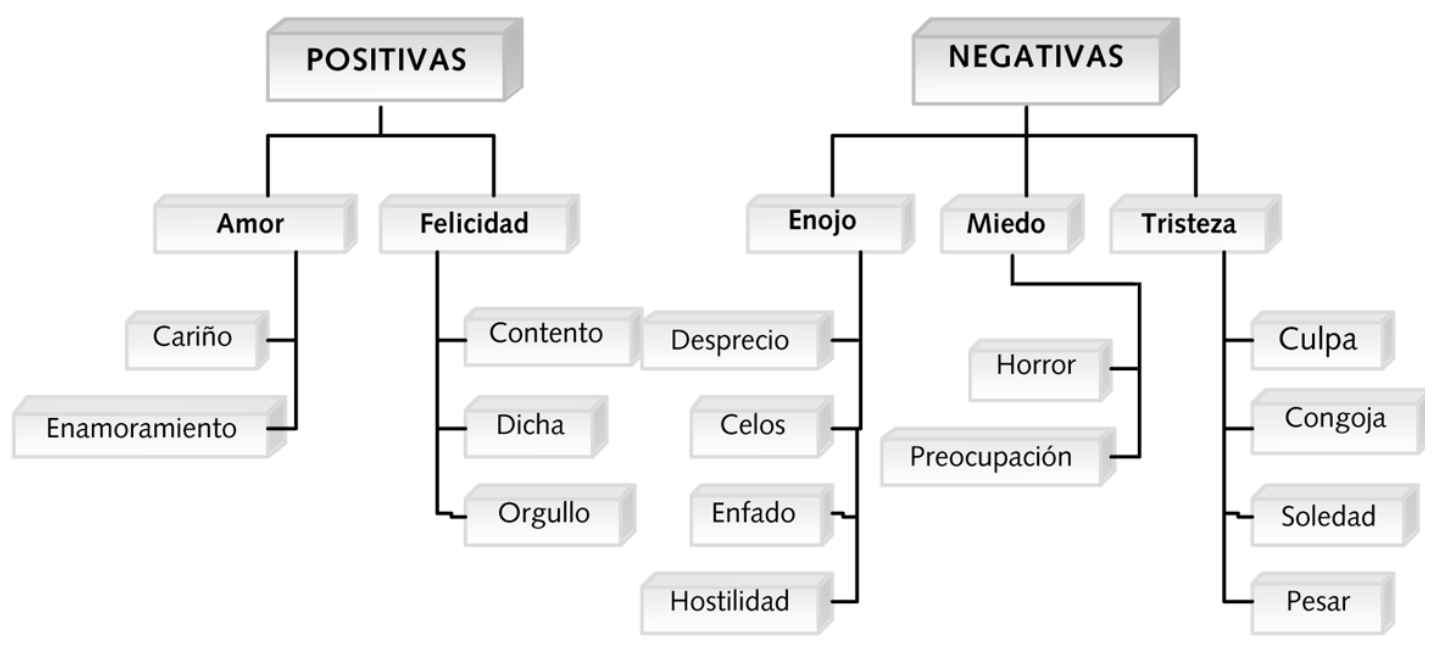

Figura 1. Categorización jerárquica de emociones positivas y negativas tanto básicas como secundarias que proponen Fischer et al. (1990 en Feldman, 2002). 
otras especies (Dylan, 2002). Si bien la experiencia favorece el desconsuelo y la angustia, existe un amplio rango de eventos que pueden detonar la emoción de miedo. De hecho, Gray (1979 en Boissy, 1995) señala que existen cinco tipos diferentes de estímulos que pueden causar esta emoción: 1) Los peligros que son parte de la historia evolutiva de la especie, 2) La novedad, 3) El aprendizaje, 4) La intensidad de un estímulo y 5) La interacción con individuos de la misma especie (conspecifics).

Ahora bien, independientemente de los eventos que lo desencadenan, el miedo es una emoción que puede ser descrita como una respuesta incluyente (Moltó, 1995 en Barrón \& Bazán, 2004; Teachman et al., 2008). En lo que atañe a lo fisiológico, la literatura señala que el miedo se traduce en una aceleración del ritmo cardiaco y la respiración, contracción muscular, temblor de piernas y manos (Lelord, 2001), aumento en la actividad del sistema neuroendócrino disparando los niveles de adrenalina y noradrenalina, incremento de la presión arterial, náuseas y sudoración (Méndez, 2006), entre otras reacciones. En cuanto a la cognición, el miedo está vinculado a la sensación de incertidumbre y la amenaza (Lazarus, 2000). Al respecto, Scherer (2001) plantea que los individuos evalúan la relevancia que tiene el acontecimiento, sus implicaciones o consecuencias, la determinación del potencial del que se dispone, para afrontar el evento y por último el significado normativo o social del mismo. En lo tocante a su manifestación explícita, el miedo es considerado como uno de los estados emocionales más expresivos (Heller, 1989 en Álvarez, 2006) evidentes en la expresión facial, las vocalizaciones (Boissy, 1995), la palidez del rostro (Berástegi, 2007; Camacho, 2002), la piloerección (Boissy, 1995; Camacho, 2002), entre otros; todas estas manifestaciones con un alto valor funcional ya que actúan como señales de alarma y constituyen las principales fuentes de comunicación social sobre algún tipo de peligro (Boissy, 1995). En lo que a las tendencias de acción del miedo se refiere, los organismos parecen tener varias opciones: paralizarse y no hacer nada (Álvarez, 2006; Camacho, 2002), escapar de ese miedo o evitarlo (Álvarez, 2006; Hamm \& Weike, 2006; Kuchel, 1991 en Boissy, 1995; Camacho, 2002) y enfrentarlo o atacar (Álvarez, 2006; Kuchel, 1991 en Boissy, 1995). La paralización es una reacción que sucede de forma involuntaria y se presenta comúnmente cuando el organismo se encuentra frente a un objeto que evalúa como terrible (Hamm y Weike, 2006), mientras que la huida y el ataque funcionan como medios de protección para mantener al organismo a salvo de aquello que lo acecha y pone en riesgo su existencia (Álvarez, 2006). Conforme la amenaza se aproxima, la intensidad de la respuesta del miedo se incrementa y con ello los recursos energéticos del organismo ya sea para escapar o (cuando no es posible escapar) quedarse para pelear (si el ataque no es la opción) o inmovilizarse completamente (inmovilización tónica). Tan pronto como la amenaza desaparece, la intensidad de la respuesta de miedo se reduce.

Resumiendo, Boissy (1995) expresa que los patrones conductuales que ejecuta un organismo ante la presencia de un objeto o situación que le produce miedo pueden llegar a ser contradictorios e ir desde la defensa activa (atacar o amenazar) hasta la evitación activa (ocultarse o huir) y la inmovilidad o inhibición del movimiento. No obstante, como señala el mismo autor, las respuestas conductuales van a depender aquí del estímulo amenazante, más concretamente si se encuentra presente (estado de miedo) o si sólo representa un peligro potencial (estado de ansiedad). Lo anterior sugiere que existe una línea muy delgada entre el miedo y otros estados afectivos (por ejemplo el temor, la angustia, la fobia, el pánico y la ansiedad) con los que frecuentemente se le confunde y con los que, al final, difiere en términos de su intensidad, duración, ambigüedad o sorpresa ante el peligro. Para esclarecer estos aspectos, dichos conceptos han sido divididos de manera general en dos grandes grupos: el del miedo y el de la angustia. Al primero pertenecen el temor, el espanto, el pavor y el terror (que a su vez se distinguen por su intensidad), mientras que la ansiedad, la melancolía y la inquietud pertenecen a la angustia. La principal diferencia entre estos dos grupos estriba en la posibilidad de controlar y conocer el peligro. En el caso de la angustia no se tiene un objeto definido que la desencadene, su objeto no tiene nombre, es ambiguo; más bien se trata de un estado de inquietud constante y, por lo tanto, se escapa del control del ser humano. Por su parte, el miedo nombra un objeto que es la fuente de peligro, ya sea latente, real o imaginaria, para de esta manera poder actuar ante ella (Méndez, 2006). 
Con lo revisado hasta el momento puede decirse entonces que el miedo es una reacción provocada por un estímulo interno o externo que es valorado como una amenaza inmediata que atenta contra el bienestar del organismo. Debido a ello se producen en éste una serie de cambios a nivel fisiológico que lo predisponen a la acción, mientras la atención se fija en la amenaza con el fin de evaluar la respuesta más apropiada. Sin embargo, cuando la activación fisiológica es muy intensa y el rendimiento del organismo se vuelve excesivo, se corre el riesgo de caer en un bloqueo emocional o de actuar de un modo ineficaz, como sucede generalmente en los ataques de pánico (Berástegi, 2007). De esta manera, el valor funcional y adaptativo que posee el miedo se pierde, convirtiéndose, como apunta Boissy (1995), en un estado emocional indeseable que puede reducir el bienestar de quien lo experimenta. De hecho, esta pérdida del control sobre la emoción del miedo ya era señalada por los griegos, quienes pensaban que este estado afectivo estaba ligado directamente a fuerzas superiores, elevado a rango de dioses $y$, por consiguiente, se escapaba del alcance del hombre (Méndez, 2006). No obstante, en la actualidad se cree que la pérdida del control sobre algunas emociones -incluida la del miedo- se debe a la incapacidad de los individuos para manejar sus emociones de manera efectiva (Goleman, 1997) o lo que es lo mismo, a un fallo en la regulación emocional (RE) (Gross y Thompson, 2007).

Así, la RE implica todos aquellos procesos extrínsecos e intrínsecos responsables del monitoreo, evaluación y modificación de reacciones emocionales, en especial de sus características intensivas y temporales, con el fin de alcanzar las propias metas (Thompson, 1994 en Gross y Thompson, 2007).

Gross (1998 en Ben-Ze'ev, 2000), por su parte, señala que la regulación o manejo emocional se refiere a cualquier iniciativa del individuo para influenciar qué emociones tener, cuándo tenerlas y cómo experimentarlas y expresarlas. Por otra parte, Snyder, Hughes y Simpson (2006) la definen como un proceso que involucra las estrategias que los individuos (solos o con relación a otros significativos) usan para influir el contenido, experiencia subjetiva y expresión de emociones tanto positivas como negativas. Así, el proceso de regulación incluye la iniciación, el mantenimiento y la expresión de emociones positivas y la evitación, minimización y enmascaramiento de las emociones negativas. Con base en estas definiciones, la RE del miedo consistiría, en general, en atenuar o incluso evitar dicho estado afectivo ante situaciones de peligro (Frijda, Manstead y Bem, 2000). Ahora la cuestión es: ¿cómo hacerlo?

Al respecto, Gross y Thompson (2007) sugieren que el individuo genera cinco procesos de RE que se traducen en una serie de estrategias clave a lo largo del proceso emocional y hacia la manifestación de la conducta. Dichos procesos son: la Selección de la Situación, la Modificación de la Situación, el Despliegue de Atención (Distracción o Concentración), el Cambio Cognoscitivo y la Modulación de la Respuesta.

- Selección de la Situación (SS). Esta pauta de RE involucra tomar acciones que aumenten la probabilidad de terminar o no en un escenario en el que se experimenten emociones, ya sean deseables o indeseables. La SS requiere del entendimiento de consecuencias probables de situaciones remotas y de respuestas emocionales esperadas con relación a éstas.

- Modificación de la Situación (MS). Se refiere a todos los esfuerzos del individuo destinados a alterar directamente la situación y el impacto emocional que ésta puede tener. Esta estrategia de RE constituye una forma potente para manejar las emociones y puede darse tanto de manera interna (cambio cognoscitivo) como externa (modificación de ambientes físicos).

- Despliegue de Atención (DA). Consiste en dirigir la atención o no dentro de una situación dada con el propósito de influenciar las emociones. De hecho, existen dos maneras básicas de dirigir la atención: a) Distracción, involucra cambiar el foco interno de la atención, tal como cuando los individuos invocan pensamientos o recuerdos que son inconsistentes con el estado emocional indeseable. b) Concentración, que implica dirigir la atención hacia las características de una situación, por ejemplo, cuando un actor trae a su mente un incidente emocional para representar esa emoción de manera convincente.

- Cambio Cognoscitivo (CC). Se refiere a cambiar la forma de apreciar la situación en la que se está para alterar su importancia, ya sea cambiando la 
manera en la que se piensa acerca de ella o modificando la propia capacidad para manejar las demandas que implica. Este elemento regulatorio sólo puede ser aplicado a la propia experiencia interna del evento y tiene que ver con la interpretación o reconstrucción que el sujeto hace de los signos fisiológicos de la excitación emocional, ya sea en una dirección positiva o negativa.

- Modulación de la Respuesta (MR). En contraste con los procesos anteriores, la modulación de la respuesta ocurre tarde en el proceso generativo de la emoción, es decir, después de que las tendencias de respuesta han sido iniciadas. De esa forma, la MR tiene que ver con la influencia que el individuo ejerce, $\tan$ directamente como le sea posible, en las respuestas fisiológicas, experienciales o conductuales de la emoción. Algunas maneras de influir en las respuestas fisiológicas son las drogas, los alimentos, el ejercicio y la relajación. Otra forma común de modulación de la respuesta involucra la regulación de la conducta expresiva en función del contexto cultural en el que se presenta, ya que hay conductas que tienen que ser moduladas en ciertos escenarios con el fin de mostrar una mayor adaptación, mientras que en otros esas mismas conductas emocionales pueden ayudar a alcanzar ciertas metas.

Este planteamiento sugiere que una emoción -ya sea positiva o negativa- se desencadena a partir de una situación relevante para el individuo que él puede elegir experimentar o no. Si la elige, tiene entonces la posibilidad de modificarla para reducir el impacto emocional de ésta. No obstante, si no la modifica pasará a centrar su atención en ella, ya sea concentrándose o distrayéndose. A continuación, el individuo evaluará la situación en función de la importancia que tenga para él, la cual puede modificar cognoscitivamente. Así, si dicha situación le resulta relevante, entonces el sujeto procederá a emitir una respuesta, que puede modular o no, y que a su vez tendrá un impacto en la situación que la originó.

Con base en la información previa y en la carencia de una medida pertinente, el propósito de este estudio fue la validación de una prueba que operacionalice los procesos de Gross y Thompson (2007) en el contexto es- pecífico del miedo para así identificar las estrategias empleadas por adultos de la Ciudad de México para enfrentarlo.

\section{MÉTODO}

\section{Participantes}

Se trabajó con una muestra no probabilística de 184 sujetos voluntarios ( 86 hombres y 98 mujeres) de la Ciudad de México con edades que oscilaban entre 18 y 36 años (con una Media de 22.4) y cuya escolaridad es de licenciatura de carreras diversas.

\section{Instrumento y Análisis}

Se utilizó la Estimación Cognoscitiva de los Procesos de Regulación Emocional (Sánchez-Aragón, 2008) que es un autorreporte dirigido a evaluar los cinco procesos de regulación emocional involucrados en la generación de estrategias de regulación de acuerdo con Gross y Thompson (2007): Selección de la Situación, Modificación de la Situación, Despliegue de Atención (con dos áreas: distracción y concentración), Cambio Cognoscitivo y Modulación de la Respuesta. Para cada sección la escala incluye reactivos con formato de respuesta tipo Likert, cuyas opciones se relacionan con grados de acuerdo (1. Totalmente en desacuerdo, 2. Desacuerdo, 3. Ni de acuerdo ni desacuerdo, 4. De acuerdo y 5 . Totalmente de acuerdo) ante reactivos que versan sobre los contenidos de cada proceso. Esta prueba fue contextualizada a la emoción del miedo para la presente investigación.

\section{Procedimiento}

Se contactó a los participantes en espacios académicos y se les pidió que contestaran la prueba, concentrándose en la emoción indicada (miedo) para poder resolver cada una de las secciones. Se les explicó que los datos se tratarían en forma confidencial y anónima y que serían analizados estadísticamente para indicar patrones generales de comportamiento.

\section{Resultados}

Para dar validez de constructo y confiabilidad a las operacionalizaciones de los cinco procesos de regulación emocional de Gross y Thompson (2007) para la emoción del miedo, se siguieron los criterios de Reyes- 
Lagunes y García y Barragán (2008) en la validación de pruebas.

Selección de la Situación (SS). En este caso, se realizó un análisis factorial de componentes principales con rotación ortogonal de donde surgió un factor con valor propio mayor a 1 que explica el $50.25 \%$ de la varianza (Tabla 1):

Tabla 1. Análisis Factorial para SS: Factor Alejamiento

\begin{tabular}{|c|c|c|c|}
\hline$\alpha=.66$ & Media $=3.05$ & $D S=.83$ & $\begin{array}{c}\text { Carga } \\
\text { Factorial }\end{array}$ \\
\hline \multicolumn{3}{|c|}{ No me acerco a ese escenario } & .787 \\
\hline \multicolumn{3}{|c|}{ Me aparto de ese contexto } & .779 \\
\hline \multicolumn{3}{|c|}{ Hago lo que está a mi alcance para evitarla } & .658 \\
\hline \multicolumn{3}{|c|}{ Hago lo que puedo para no enfrentarla } & .592 \\
\hline
\end{tabular}

Modificación de la Situación (MS). En este rubro se realizaron los mismos procedimientos estadísticos y se obtuvieron dos factores con valor propio mayor a 1 , los cuales explican el $52.71 \%$ de la varianza; no obstante, el segundo no mostró un coeficiente de confiabílidad mínimo de .60 , por lo cual no es reportado (Tabla 2):

Tabla 2. Factor para MS: Alteración del Evento

\begin{tabular}{|c|c|c|c|}
\hline$\alpha=.60$ & Media $=3.37$ & $\mathrm{DS}=.82$ & $\begin{array}{c}\text { Carga } \\
\text { Factorial }\end{array}$ \\
\hline \multicolumn{3}{|c|}{ Me esfuerzo para cambiar el matiz de las cosas } & .772 \\
\hline \multicolumn{3}{|c|}{ Hago lo posible por modificar la situación } & .755 \\
\hline \multicolumn{3}{|c|}{ Cambio mi actitud para modificarla } & .650 \\
\hline
\end{tabular}

Despliegue de Atención (DA). En esta sección se realizaron tres análisis factoriales de componentes principales con rotación ortogonal: uno para distracción, otro para concentración y otro para aquellos reactivos citados en la literatura como parte definitoria de este proceso. En el caso de la distracción se obtuvo un solo factor que explica el $38.64 \%$ de la varianza, y lo mismo sucedió para la concentración, lo cual explica el $59.80 \%$ y para la teoría se obtuvo un factor que explica el 49.22\% (Tabla 3):
Tabla 3. Factores encontrados para DA

\begin{tabular}{|c|c|c|c|}
\hline Distracción $=.60^{1}$ & Media $=3.07$ & $\mathrm{DS}=.73$ & $\begin{array}{l}\text { Carga } \\
\text { Factorial }\end{array}$ \\
\hline \multicolumn{3}{|c|}{$\begin{array}{l}\text { Pienso en otras cosas para no involucrarme en } \\
\text { esa situación }\end{array}$} & .729 \\
\hline \multicolumn{3}{|c|}{ Me enfoco en otra cosa } & .702 \\
\hline \multicolumn{3}{|c|}{ Pienso en algo opuesto a la emoción } & .698 \\
\hline \multicolumn{3}{|c|}{$\begin{array}{l}\text { Me acuerdo de situaciones diferentes a las } \\
\text { que estoy viviendo }\end{array}$} & .497 \\
\hline Concentración $=.60$ & Media $=2.99$ & $\mathrm{DS}=.74$ & $\begin{array}{l}\text { Carga } \\
\text { Factorial }\end{array}$ \\
\hline \multicolumn{3}{|c|}{ Suelo ensimismarme con lo que estoy sintiendo } & .720 \\
\hline \multicolumn{3}{|c|}{ Me "clavo" en la experiencia emocional } & .646 \\
\hline \multicolumn{3}{|c|}{ Reflexiono sobre la situación actual } & .612 \\
\hline \multicolumn{3}{|c|}{$\begin{array}{l}\text { Me concentro en las sensaciones que } \\
\text { me despierta }\end{array}$} & .586 \\
\hline Negación= .66 & Media $=2.10$ & $\mathrm{DS}=.77$ & $\begin{array}{c}\text { Carga } \\
\text { Factorial }\end{array}$ \\
\hline \multicolumn{3}{|c|}{$\begin{array}{l}\text { Me tapo los oídos para no darme cuenta } \\
\text { de las cosas }\end{array}$} & .776 \\
\hline \multicolumn{3}{|c|}{ Me cubro los ojos para no ver lo que pasa } & .723 \\
\hline \multicolumn{3}{|l|}{ No escucho } & .666 \\
\hline \multicolumn{3}{|l|}{ No le presto atención } & .633 \\
\hline
\end{tabular}

Cambio Cognoscitivo (CC). Al respecto, el análisis factorial de componentes principales con rotación ortogonal reportó dos factores con valor propio mayor a 1 que explican el $50.61 \%$ de la varianza, sin embargo, únicamente el primero obtuvo un coeficiente de confiabilidad considerable (Tabla 4):

Tabla 4. Análisis Factorial para CC: Evaluación de Recursos

\begin{tabular}{|c|c|c|c|}
\hline$\alpha=.60$ & Media=3.46 & $\mathrm{DS}=.81$ & $\begin{array}{c}\text { Carga } \\
\text { Cognoscitiva }\end{array}$ \\
\hline \multicolumn{3}{|c|}{ Reconsidero el valor de la situación } & .799 \\
\hline \multicolumn{3}{|c|}{$\begin{array}{l}\text { Evalúo el impacto que tiene la emoción } \\
\text { en mi vida }\end{array}$} & .702 \\
\hline \multicolumn{3}{|c|}{$\begin{array}{l}\text { Reflexiono sobre la capacidad que tengo } \\
\text { para manejarla }\end{array}$} & .671 \\
\hline
\end{tabular}

${ }^{1}$ En este proceso como en el de Modulación de la Respuesta sólo se incluyen cuatro reactivos como ejemplos. 
Modulación de la Respuesta (MR). Para este proceso se realizó el análisis factorial pertinente (de componentes principales con rotación ortogonal), el cual arrojó 7 factores que explican el $48.32 \%$ de la varianza. Después se obtuvieron sus coeficientes de confiabilidad Alpha de Cronbach observándose coeficientes robustos en seis de ellos (Tabla 5):

Tabla 5. Análisis Factorial del Proceso MR

\begin{tabular}{|c|c|c|c|}
\hline Reflexión-Calma $\alpha=.78$ & Media=3.55 & $\mathrm{DS}=.69$ & Carga Factorial \\
\hline \multicolumn{3}{|l|}{ Reflexiono } & .719 \\
\hline \multicolumn{3}{|l|}{ Busco la razón de mi emoción } & .705 \\
\hline \multicolumn{3}{|l|}{ Trato de tranquilizarme } & .613 \\
\hline \multicolumn{3}{|c|}{ Pienso en muchas formas para salir de esto } & .497 \\
\hline Búsqueda de apoyo $\alpha=.75$ & Media=3.09 & $\mathrm{DS}=.72$ & Carga Factorial \\
\hline \multicolumn{3}{|l|}{ Lo comparto } & .787 \\
\hline \multicolumn{3}{|l|}{ Busco refugio en alguien } & .709 \\
\hline \multicolumn{3}{|l|}{ Busco apoyo } & .649 \\
\hline \multicolumn{3}{|c|}{ Le hablo a mi mejor amigo (a) para desahogarme } & .578 \\
\hline Agotamiento $\alpha=.73$ & Media $=2.35$ & $\mathrm{DS}=.73$ & Carga Factorial \\
\hline \multicolumn{3}{|l|}{ Me siento cansado } & .687 \\
\hline \multicolumn{3}{|l|}{ Me duermo } & .521 \\
\hline \multicolumn{3}{|l|}{ Me dan ganas de comer } & .515 \\
\hline \multicolumn{3}{|l|}{ Me aíslo } & .513 \\
\hline Agresión $\alpha=.72$ & Media $=2.44$ & $\mathrm{DS}=.88$ & Carga Factorial \\
\hline \multicolumn{3}{|l|}{ Hablo fuerte } & .704 \\
\hline \multicolumn{3}{|l|}{ Ofendo } & .686 \\
\hline \multicolumn{3}{|l|}{ Exploto } & .667 \\
\hline \multicolumn{3}{|l|}{ Discuto con los demás } & .609 \\
\hline Excitabilidad $\alpha=.69$ & Media $=2.61$ & $\mathrm{DS}=.70$ & Carga Factorial \\
\hline \multicolumn{3}{|l|}{ Me pongo nervioso } & .476 \\
\hline \multicolumn{3}{|l|}{ Tiemblo } & .735 \\
\hline \multicolumn{3}{|l|}{ Me escondo } & .695 \\
\hline \multicolumn{3}{|l|}{ Me congelo totalmente } & .583 \\
\hline Superación $\alpha=.67$ & Media $=2.67$ & $\mathrm{DS}=.70$ & Carga Factorial \\
\hline \multicolumn{3}{|l|}{ Me siento relajado } & .676 \\
\hline \multicolumn{3}{|c|}{ Disfruto y aprendo a gozar esta emoción para tratar de no repetirla } & .634 \\
\hline \multicolumn{3}{|l|}{ Veo las cosas objetivamente } & .507 \\
\hline \multicolumn{3}{|l|}{ Sigo con mi vida normal } & .395 \\
\hline
\end{tabular}




\section{DISCUSIÓN}

En primera instancia, la revisión teórica sobre las funciones adaptativas del miedo plantea la necesidad de regular dicho estado emocional tanto a nivel fisiológico como cognoscitivo, expresivo, conductual y experiencial con el propósito de incrementar la probabilidad de éxito del individuo al enfrentarse a una situación que lo detona. Por ello, la propuesta de Gross y Thompson (2007) sobre los mecanismos o estrategias para lograr una regulación emocional exitosa en términos instrumentales y afectivos se convierte en una promesa de entendimiento de los procesos cognoscitivos y conductuales involucrados en la emoción del miedo en particular. Así, Sánchez-Aragón (2008) operacionaliza los cinco procesos de RE y en este trabajo son aplicados al contexto emocional del Miedo. Los resultados por proceso señalan lo siguiente:

Selección de la Situación. En este caso, se obtuvo un factor denominado Alejamiento que incluye cuatro reactivos que versan sobre la disposición inicial del individuo para apartarse de situaciones que le pueden producir miedo, lo cual es fundamentado por la literatura (Álvarez, 2006; Hamm \& Weike, 2006; Kuchel, 1991 en Boissy, 1995; Camacho, 2002; Märtin \& Boeck, 1998; Wulkimir, 1967 en Goleman, 1997) la cual señala que la evitación, la huida o el alejamiento es una de las tendencias conductuales básicas de los individuos al experimentar emociones negativas, particularmente la de miedo.

Cabe señalar que esta tendencia se presenta antes de que las personas estén experimentado la emoción, es decir, se anticipan y eligen alejarse de la fuente de miedo. Esto debido a que según Gross y Thompson (2007), el individuo realiza una evaluación de las posibles consecuencias de situaciones que pueden producir dicha emoción. O bien, de acuerdo con Scherer (2001), ésta reacción puede darse en función de la relevancia que el acontecimiento tiene para la persona, la evaluación de las implicaciones o consecuencias de este, la determinación del potencial del que se dispone para afrontar el evento y, por último, la evaluación del significado normativo o social.

Modificación de la Situación. En este rubro se obtuvo también un factor consistente que se denominó Alteración del Evento, porque incluye reactivos que reflejan los esfuerzos del individuo por cambiar la situación a nivel interno con el propósito de reducir el impacto emocional que el miedo genera (Gross \& Thompson, 2007). Ahora bien, dichos esfuerzos para modificar la situación, de acuerdo con Märtin y Boeck (1998), son concebidos por el propio organismo ante la experiencia de emociones negativas, dentro de las cuales Fischer et al. (1990 en Feldman, 2002) sitúan al miedo.

- Despliegue de Atención. En esta sección se obtuvieron tres factores o estrategias en forma separada.

- Distracción. Es un factor que incluye cinco reactivos que estiman la disposición del individuo para dirigir su atención a cualquier otra cosa que no sea el estímulo desencadenador de la emoción de miedo.

- Concentración. Es un factor que tiene cinco reactivos que versan sobre el ensimismamiento del individuo ante la experiencia emocional de miedo, ya sea dándole vueltas a la situación una y otra vez o prestándole demasiada atención a la vivencia del momento.

- Negación. Es un factor que se compone por cuatro reactivos en los que se evidencian algunos de los medios básicos que utilizan los individuos con el fin de atenuar o no engancharse en la experiencia emocional de miedo.

Como puede apreciarse, los factores de Distracción y Concentración -en este caso, ante un estimulo generador de miedo- coinciden con las estrategias de regulación emocional planteadas por Gross y Thompson (2007) para el Despliegue de Atención. En cambio, el factor de Negación, es decir, cerrarse ante la percepción de algún estímulo o evento que puede detonar la emoción de miedo, aparece aquí como una forma alternativa e ingenua en el repertorio de las personas para manejar las situaciones.

De esta manera, como Berástegi (2007) señala, aunque en determinado momento durante la experiencia de miedo es necesario fijarse y mantener la atención (concentrarse) en la amenaza con el fin de evaluar la respuesta más apropiada, para las personas en algunos casos es mejor desviar dicha atención (distracción, negación) con el propósito de reducir la in- 
tensidad y percepción de peligro ante dicha experiencia emocional.

Cambio Cognoscitivo. Con respecto a este proceso, se encontró un factor al que se nombró Evaluación de Recursos, el cual posee tres reactivos que versan sobre la apreciación de una situación-estímulo (que en este caso genera la emoción de miedo) y la valoración del alcance y la capacidad que tiene la persona para reaccionar ante ésta. Scherer (2001) incluye ambos elementos (junto con la evaluación de las implicaciones o consecuencias de un evento y la evaluación del significado normativo o social de éste) en los procesos evaluativos que surgen de la estimación de un mismo evento -en este caso, un estímulo amenazante o peligroso-.

Asimismo, cabe señalar la relevancia de la Evaluación de Recursos en la generación del estado emocional de miedo ya que la cognición es un elemento necesario y suficiente para que una emoción se desencadene (Lazarus, 1991), pero también para que ésta sea regulada. Y es que, como sugieren Gross y Thompson (2007), las valoraciones que haga el individuo pueden llevarlo a interpretar o reconstruir los signos fisiológicos de la excitación emocional ya sea en una dirección positiva o negativa y con base en ello mejorar o empeorar las capacidades necesarias para manejar las demandas que la situación exige.

Modulación de la Respuesta. Para este proceso los factores obtenidos se reflejan en las variadas respuestas que los individuos pueden presentar ante el miedo -a excepción del factor excitabilidad- y que ocurren ya tarde en el proceso generativo de la emoción, es decir, después de que las tendencias de respuesta (evitación, enfrentamiento o paralización) han sido iniciadas, de ahí que precisamente pertenezcan a la dimensión de modulación de la respuesta (Gross \& Thompson, 2007). Los factores que componen este proceso, entonces, se refieren a las formas o estrategias que las personas -al experimentar miedo- ejecutan con el fin de regular su emoción.

El factor de Reflexión-Calma, se define por el proceso de especulación que la persona lleva a cabo con el fin de encontrar la razón o causa de la emoción para así evaluar la magnitud de la amenaza, estimar las posibles salidas, enfrentarla en caso de ser posible y con ello lograr la tranquilidad. Al respecto, Scherer (2001) plantea que los individuos realizan varios procesos evaluativos que se encargan de recoger información sobre el nivel de peligro del estímulo para así detectar la relevancia que tiene el acontecimiento, la evaluación de las implicaciones o consecuencias de éste, la determinación del potencial de afrontamiento del que se dispone ante ese evento, y, por último, la evaluación del significado normativo o social del mismo. El factor de Búsqueda de Apoyo se refiere a compartir la emoción buscando refugio y apoyo en alguien con quien se puede llorar, un amigo con quien desahogarse a la vez que la persona se aleja de la fuente de la emoción. Con referencia a esto, Cienfuegos (2004) señala que cuando una persona se encuentra en un momento de necesidad (como puede ser al sentir miedo) se busca ayuda accesible en personas, grupos e instituciones con los cuales el individuo percibe un soporte emocional, instrumental y de comunicación, lo cual le brinda oportunidades para sentirse mejor y para poder re-ajustarse a su medio.

El factor de Agotamiento versa sobre las sensaciones de cansancio, aletargamiento, pérdida y ganancia de apetito, búsqueda del aislamiento para escribir sobre la emoción experimentada y autocompadecerse. Esto conforma una forma de reaccionar no citada en la literatura, pero parecería que emerge como una forma de evasión de la realidad que la persona experimenta o bien una manifestación de la incapacidad de responder efectivamente ante ella. El factor de Agresión involucra el hablar fuerte, ofender, y explotar en presencia de los demás generando discusiones. De acuerdo con algunos autores (por ejemplo Álvarez, 2006 y Kuchel, 1991 en Boissy, 1995), éstas son reacciones de ataque que funcionan como medios de protección para mantener al organismo a salvo de aquello que lo acecha y pone en riesgo su existencia.

El factor de Excitabilidad se refiere a que la persona, al sentir miedo, se pone nerviosa, aprieta cualquier cosa que tiene enfrente, tiembla, se esconde, se congela y reza. Este factor incluye elementos de dos tipos: por un lado están las reacciones fisiológicas que Lelord (2001) señala como parte del miedo y que aumentan la actividad del sistema neuroendócrino. Éstas consisten en que se disparan los niveles de adrenalina y noradrenalina, hay un incremento en la presión arterial que provoca náuseas y sudoración (Méndez, 
2006), entre otras reacciones para activar la respuesta abierta a la emoción. Y por otro lado, el bloqueo psicológico que se presesenta como una respuesta natural ante la experiencia ya que, como mencionan Álvarez (2006) y Camacho (2002): la paralización es una reacción que ocurre de forma involuntaria y se presenta comúnmente cuando el organismo se encuentra frente a un objeto que evalúa como terrible. El factor de Superación implica el disfrutar, sentirse relajado, aprender a sufrir y gozar los momentos posteriores a la emoción pero deseando no repetirla y siendo más objetivo, para así continuar con la vida normal. En lo que toca a este factor, Hamm y Weike (2006) plantean que la activación del sistema del miedo se incrementa conforme se aproxima la amenaza o el estímulo intimidante, generando respuestas de escape, pelea o inmovilización, pero tan pronto como la amenaza desaparece, la intensidad de la respuesta de miedo se reduce.

Por último, los resultados de esta investigación arrojan que los individuos pueden influir en el curso de su miedo de diferentes maneras a lo largo del proceso generativo de dicha emoción. Así, antes de sentir esta emoción los individuos pueden elegir evitar estar en situaciones o ante estímulos que detonen esta experiencia (Me aparto de ese contexto). Si no lo hacen y ya están en ella, tienen la posibilidad entonces de modificarla cognoscitivamente (Me esfuerzo para cambiar el matiz de las cosas) para reducir su impacto emocional.

\section{REFERENCIAS}

Álvarez, E. (2006). El miedo manejado como placer estético en el cine de terror (El vampiro: su principal personaje). Tesis de Licenciatura no publicada, Universidad Nacional Autónoma de México.

Barrón, J. Z. \& Bazán, A. (2004). Expresión de sentimientos en el género masculino. Tesis de Licenciatura no publicada, Universidad Nacional Autónoma de México.

Ben-Ze'ev, A. (2000). The subtlety of emotions. Massachussetts, E.E. U.U.: Massachussetts Institute of Technology.

Berástegi, J. (2007). Regulación emocional: El Miedo. Recuperado el 7 de julio de 2008 de $h t t p: / / b l o g . e i t b 24$. com/inteligenciaemocional/2007/10/15/regulacionemocional-el-miedo/.
No obstante, si no modifican la experiencia, ésta empezará a movilizar los procesos intencionales para regularla, ya sea concentrándose en ella (Me "clavo" en la experiencia emocional), distrayéndose (Pienso en otras cosas para no involucrarme en esa situación) o negándola (Me tapo los oídos para no darme cuenta de las cosas). Si esto no funciona, el individuo puede tratar de generar un cambio cognoscitivo a través de la evaluación de la situación (Re-considero el valor de la situación) para de esta manera replantear la relevancia que ésta tiene para él. Si aún así la situación resulta ser de suma importancia para la persona, entonces procederá a emitir una respuesta (huir, atacar o paralizarse) que puede modular utilizando las siguientes estrategias: reflexionar-calmarse, buscar apoyo, tratar de reducir su actividad física (agotamiento) y fisiológica (excitabilidad), entre otras.

La Regulación Emocional (RE) del miedo en adultos mexicanos está en sus inicios, y la identificación de sus estrategias representa un primer paso en el conocimiento de las respuestas más instintivas o inmediatas a su experiencia para así poder trabajar con aquellas que no son saludables para el individuo. Asimismo, se perfila la posibilidad de conocer aspectos de la cultura, la personalidad, de los propios recursos de las personas en el manejo adecuado de esta emoción así como sus consecuencias en la percepción de utilidad y del bienestar subjetivo de cada persona.

Boissy, A. (1995). Fear and fearfulness in animals. The Quarterly Review of Biology, 70, 2, 165-191.

Camacho, C. (2002). Implicaciones de las emociones en la construcción de enfermedades psicosomáticas. Tesis de Licenciatura no publicada, Universidad Nacional Autónoma de México.

Cienfuegos, Y. I. (2004). Evaluación del conflicto, satisfacción marital y apoyo social en mujeres violentadas: un estudio comparativo. Tesis de Licenciatura no publicada, Universidad Nacional Autónoma de México.

Dylan, E. (2002). Emoción: La ciencia del sentimiento. Madrid: Taurus.

Feldman, R. S. (2002). Psicología con aplicaciones en países de habla hispana. (4ª ed.). México: McGraw-Hill. 
Frijda, N. H., Manstead, A. S. R. \& Bem, S. (2000). Influence of emotion on beliefs. En N. H. Frijda, A. S. R. Manstead \& S. Bem. (Eds.). Emotions and beliefs: how feelings influence thoughts. Reino Unido: Cambridge University Press.

Goleman, D. (1997). Salud Emocional. Barcelona: Kairós.

Gross, J. J. \& Thompson, R. A. (2007). Emotion regulation: Conceptual foundations. En J. J. Gross (ed.). Handbook of emotion regulation (pp. 3-24). New York: The Guilford Press.

Hamm, A. O. \& Weike, A. I. (2006). The neuropsychology of fear learning and fear regulation. International Journal of Psychophysiology, 60, 3, 349-350.

Lazarus, R. S. (2000). Estrés y emoción: manejo e implicaciones en nuestra salud. Bilbao: Desclée de Brouwer.

Lelord, F. (2001). La fuerza de las emociones. Barcelona: Kairós.

Märtin, D. \& Boeck, D. (1998). ¿Qué es la inteligencia emocional? España: EDAF.

Méndez, C. (2006). El miedo al delito violento como un factor en la construcción del lugar. Estudio comparativo de dos centros comerciales en el D.F. 1999-2004. Tesis de licenciatura no publicada, Universidad Nacional Autónoma de México.

Reidl-Martínez, L. (2005). Celos y envidia: emociones humanas. México: Universidad Nacional Autónoma de México.
Reyes Lagunes, I. \& García y Barragán, L. F. (2008). Hacia un procedimiento de validación psicométrica culturalmente relevante. En S. Rivera Aragón, R. Díaz Loving, R. Sánchez Aragón e I. Reyes Lagunes (Eds.). La Psicología Social en México (pp. 625-630). México: AMEPSO.

Sánchez-Aragón, R. (2007). Pasión romántica: Más allá de la intuición una ciencia del amor. México: Miguel Ángel Porrúa.

Sánchez-Aragón, R. (2008). Propuesta de estimación cognoscitiva de la regulación emocional y predicción en emociones básicas. Manuscrito no publicado.

Scherer, K. R. (2001). Appraisal considered as a process of multilevel sequential checking. En K. R. Scherer, A. Schorr \& T. Johnstone (Eds.). Appraisal processes in emotion: Theory,_methods, research. (pp. 92-120). New York: Oxford University Press.

Snyder, D. K., Simpson, J. A., \& Hughes J. N. (2006). Emotion regulation in couples and families. Washington, D. C.: American Psychological Association.

Teachman, B. A., Stefanucci, J. K., Clerkin, E. M., Cody, M. W. \& Proffitt, D. R. (2008). A new mode of fear expression: Perceptual bias in height fear. Emotion, 8 , 2, 296-301. 